\title{
Epithelioid Haemangioendothelioma of the Mesentery
}

\author{
Oluwole O. P. ${ }^{1}$, Abimiku B. A. ${ }^{1}$, Sani A. S. ${ }^{2}$ and Ogbo O. F. ${ }^{2}$ \\ 1. Department of Pathology/Forensic Medicine, University of Abuja/Department of Histopathology, University of Abuja Teaching \\ Hospital, Gwagwalada, Abuja 900001, Nigeria \\ 2. Division of General Surgery, Department of Surgery, University of Abuja Teaching Hospital, Gwagwalada, Abuja 900001, \\ Nigeria
}

\begin{abstract}
Epithelioid haemangioendothelioma is a rare vascular tumor, described for the first time in 1975 by Dail and Liebow as an aggressive bronchoalveolar cell carcinoma. It represents $1 \%$ of all vascular tumours, due to its heterogeneous presentation, it is often misdiagnosed and not suitably treated. The etiology is still a dilemma. Most of the times it affects lung, liver and bones, although this kind of tumor may involve the head and neck area, breast, lymph nodes, mediastinum, brain and meninges, the spine, skin, abdomen and many other sites. Mesentery involvement is very rare. We report a case of 22-year old female student, who presented with recurrent abdominal pain of four month duration and one month duration of abdominal swelling that was later histopathological diagnosed to be epithelioid haemangioendothelioma.
\end{abstract}

Key words: Epithelioid haemangioendothelioma, mesentery, young female student.

\section{Introduction}

Epithelioid haemangioendothelioma (EHE) is a rare vascular tumor with an epithelioid and histiocytoid appearance, originating from vascular endothelial or pre-endothelial cells. It represents less than $1 \%$ of all vascular tumors and was described for the first time in 1975 by Dail and Liebow as pulmonary EHE (P-EHE) [1]. The term epithelioid haemangioendothelioma was introduced in 1982 by Weiss and Enzinger to describe a vascular tumor of bone and soft tissue showing features between hemangioma and angiosarcoma [2,3]. Corrin et al. [4] demonstrated the presence of tumor cells deriving from a lineage capable of differentiation along endothelial lines by using immunohistochemical techniques. Later, Weldon-Linne et al. [5] confirmed these findings using electron microscopy and revealed a diffuse cytoplasmic staining of the malignant cells with a factor VIII-related antigen.

This tumor has been encountered in all age groups, but it is most common in the fourth through seventh

Corresponding author: Oluwole O. P., M.B.B.S, FMCPath (Nigeria), associate professor, research fields: genitourinary pathology with bias towards prostate cancer, anorectal and breast carcinoma. decades of life. Overall, EH has an equal sex distribution, although cases presenting in the lungs and liver are more frequent in women [6]. There is virtually no site which is immune to the occurrence of an $\mathrm{EH}$ [7-10]. The intermediate grade of this tumor does not belie its capacity for local recurrence and even distant metastasis. The overall mortality rate for $\mathrm{EH}$ is only about $13 \%$, but is as high as $65 \%$ for tumors arising in the liver [11].

This report documents our experience with one of the least common sites of EHE, namely the mesentery surrounding the caecum and appendix without a definable origin in any of the abdominal organs. We demonstrate that the light microscopic features are very similar to EHE arising at more conventional sites such as the liver, that immunohistochemical analysis provides a reliable approach for confirming or establishing the diagnosis, and that at least 1 endothelial marker (either CD31, CD34, or factor VIII) should be positive for a definitive diagnosis [6].

We report a case of 22-year old female student, who presented with recurrent abdominal pain of four month duration and one month duration of abdominal 
swelling that was later histopathological diagnosed to be epithelioid haemangioendothelioma.

\section{Case Report}

A 22-year old female student who presented with recurrent abdominal pain of 4 months duration and abdominal swelling of one month duration to Gynaecology clinic.

She developed recurrent abdominal pain at the right iliac fossa region. The pain was colicky, non-radiating and severe enough to stop her from doing her chores but relieved by analgesics. The current episode started one month prior to presentation.

Also noticed swelling in the right iliac fossa which rapidly increased to the size of a twenty-two weeks gravid uterus. There is associated nausea, vomiting, change in bowel habit; constipation alternating with diarrhoea. There was no passage of melena stools or haematochezia.

There is also an associated high grade fever, with no associated chills or rigor. No vaginal discharge or bleeding per vaginum.

She had termination of pregnancy nine (9) months prior to presentation.

She neither drink alcohol nor smoke cigarette.

Physical examination revealed a young lady chronically ill-looking, febrile $\left(\mathrm{T}-39^{\circ} \mathrm{C}\right)$, pale, anicteric and acyanosed.

The chest was symmetrical with respiratory rate of 24 cycles/min. The pulse rate was 120 beats/min, regular and of moderate volume. BP was 120/70 $\mathrm{mmHg}$ sitting.

The abdomen was scaphoid with a right iliac fossa mass, which was tender, smooth surfaced and with cystic and solid areas, measures $10 \mathrm{~cm}$ by $12 \mathrm{~cm}$ in size. Percussion note over the mass was dull. The liver spleen and kidneys were not palpably enlarged.

The haemogram showed haemoglobin of $7.9 \mathrm{~g} / \mathrm{dl}$. WBC of $6.8 \times 10^{9} / \mathrm{L}$, platelet of $274 \times 10^{9} / \mathrm{L}$, hypochromasia, leukocytosis with toxic granulation and left shift. Human immunodeficiency virus (HIV) screen, serum HCG and Urinalysis were negative. Liver function test, Urea, electrolyte and creatinine were not contributory. The abdominal ultrasound scan showed right ovarian mass with homogenous collection.

A provisional diagnosis of right tubo-ovarian abscess was made. She was resuscitated and planned for exploratory laparotomy and surgeons where invited at surgery due to suspicion of bowel involvement. Intra-operatively, a huge highly vascular tumour involving the ileum, caecum, appendix and mesentery having cystic and solid areas was resected. She had right hemi colectomy with jejuno-colic anastomosis.

The patient made an uneventful post-operative recovery and was discharged after ten (10) days.

The gross finding is that of a huge cystic tumour measuring $15 \times 10 \times 8 \mathrm{~cm}$ and weighing $400 \mathrm{~g}$. Cut section shows a variegated tumour with areas of cystic degeneration containing serosanguinous fluid (Fig. 1).

The microscopic features show an intermediated vascular tumour growing in sheets around blood vessels. It is composed of spindle cells having moderately pleomorphic hyperchromatic to vesicular nuclei with prominent nucleoli and amphophilic cytoplasm. There are mucin-like intracytoplasmic vacuolations within the tumour cells, histiocytoid cells and osteoclast-like multinucleated giant cells (Fig. 2).

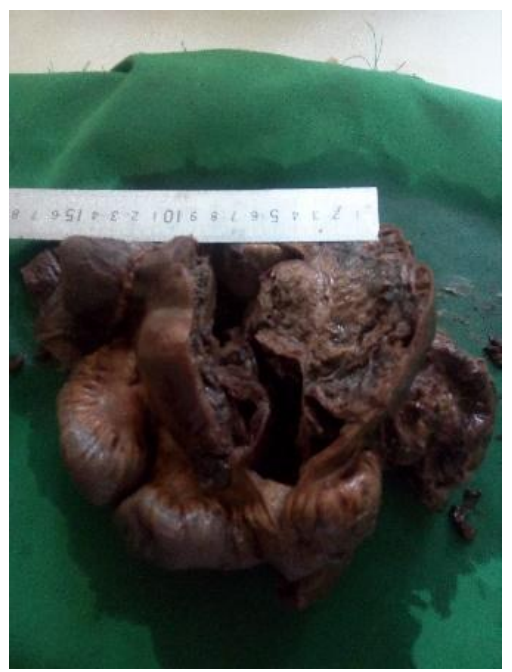

Fig. 1 Gross finding showing the tumour attached to the mesentery of the right hemicolectomy specimen. 


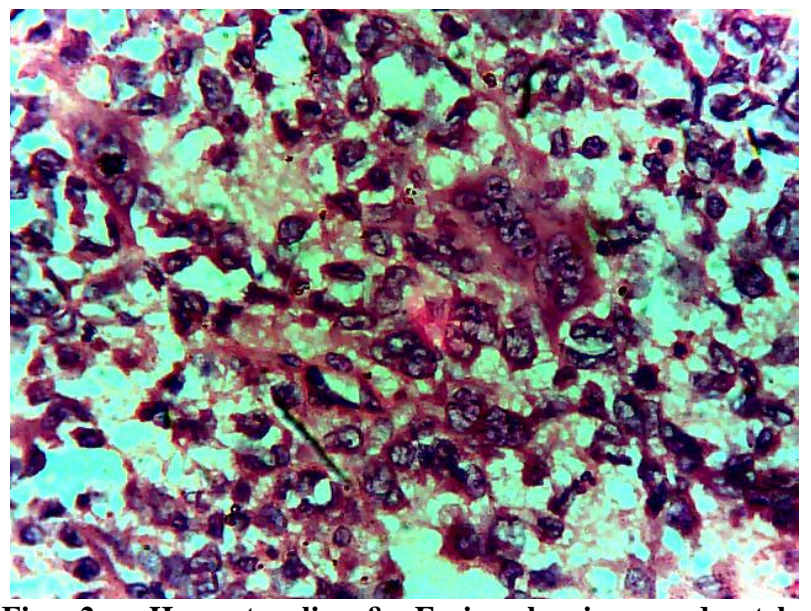

Fig. 2 Haematoxylin \& Eosin showing moderately pleomorphic hyperchromatic vesicular nuclei with prominent nucleoli, intracytoplasmic vacuolations and multinucleated giant cells. $\times \mathbf{1 0 0}$.

\section{Discussion}

Epithelioid haemangioendothelioma (EHE) is a rare low grade malignant vascular tumor which was first described by Weiss and Enzinger in 1982 [1, 2]. It has prevalence of 1 per 100,000 in the general population [12]. The etiology is still a dilemma. Studies about suggestive hypothesis are ongoing. Most of the times it affects lung, liver and bones, although this kind of tumor may involve the head and neck area, breast, lymph nodes, mediastinum, brain and meninges, the spine, skin, abdomen and many other sites [12].

The liver is one of the better-documented primary sites of EHE, likely due in part to the fact that hepatic transplantation is the treatment of choice, and identification of EHE in the lung addressed the question of the nature of the so-called intravascular bronchio-alveolar tumor [11].

There are no reports on the occurrence of this tumour in the mesentery making our patient an index case. The principal symptoms of the patient are abdominal pain and right iliac fossa swelling that kept on increasing in size, these symptoms are similar to those reported by Posligua et al. [6] and Hamirani et al. [13] in their studies on primary peritoneal epithelioid haemangioendothelioma.

The microscopic finding is consistent with epithelioid haemangioendothelioma, but differential diagnosis include metastatic carcinoma as well as mesenchymal tumours with epithelioid features like epithelioid angiosarcoma. However, the low mitotic activity (< 5/50 HPF), minimal pleomorphism, lack of necrosis and the presence of intracytoplasmic vascular lumen formation should distinguish epithelioid haemangioendothelioma from epithelioid angiosarcoma [14].

Immunohistochemistry is an essential adjunct for differentiation EHE from morphologic pretenders, including carcinoma, epithelioid leiomyosarcoma, and malignant gastrointestinal stromal tumor with epithelioid features [6]. The following vascular markers of CD31, CD34, S-100, Vimentin and factor VIII-antigen, as well as thrombomodulin, differentiate EHE from the other tumor types [6]. Although Immunohistochemistry is essential in characterizing the tumour and in differential diagnosis, but Zarifa and Kemal [15] did not find immunohistochemistry that useful due non-specificity of these marker as some are also expressed by benign vascular tumours.

Our patient also was thought as probably benign tumor and so underwent local excision only. She is currently free from recurrence for 6 months. We will continuously follow up and observe our patient to ensure that there is no recurrence of the tumour due to its malignant potential.

The role of adjuvant chemotherapy remains unclear. Läuffer et al. [16] and Makhlouf et al. [17] performed adjuvant chemotherapy with hepatic intra-arterial 5-fluorouracil, radiotherapy, and/or treatment with interferon- $\alpha-2$ in cases of epithelioid haemangioendothelioma of the liver. However, the result remained unclear, since the small number of treated cases and the unpredictable evolution of epithelioid haemangioendothelioma does not permit conclusions to be drawn $[16,17]$.

In conclusion, epithelioid haemangioendothelioma is a very rare mesenchymal tumor as primary site of mesentery, however, with a high index of suspicion, 
good clinical acumen with prompt surgical and meticulous histopathological diagnosis it can be diagnosed.

\section{References}

[1] Dail, D. H., and Liebow, A. A. 1975. "Intravascular Bronchioloalveolar Tumor.” Am. J. Pathol. 78 (1): 6a-7a.

[2] Weiss, S. W., and Enzinger, F. M. 1982. "Epithelioid Haemangioendothelioma: A Vascular Tumor Often Mistaken for a Carcinoma." Cancer 50 (1): 970-81.

[3] Weiss, S. W., Ishak, K. G., Dail, D. H., Sweet, D. E., and Enzinger, F. $\quad$ M. $1986 . \quad$ "Epithelioid Haemangioendothelioma and Related Lesions." Semin. Diagn. Pathol. 3 (1): 259-87.

[4] Corrin, B., Manners, B., Weaver, L., and Millard, M. 1979. "Histogenesis of the So-Called 'Intravascular Bronchioloalveolar Tumor'.” J. Pathol. 123 (1): 163-7.

[5] Weldon-Linne, C. M., Victor, T. A., Fry, W. A., and Christ, M. L. 1981. "Angiogenic Nature of the Intravascular Bronchioloalveolar Tumor of the Lung: An Electron Microscopic Study." Arch. Pathol. 105 (2): 174-9.

[6] Posligua, L., Anatelli, F., Dehner, L. P., and Pfeifer, J. D. 2006. "Primary Peritoneal Epithelioid Haemangioendothelioma." International Journal of Surgical Pathology 14 (3): 257-67.

[7] Baehring, J. M., Dickey, P. S., and Bannykh, S. I. 2004. "Epithelioid Haemangioendothelioma of the Suprasellar Area: A Case Report and Review of the Literature." Arch. Pathol. Lab. Med. 128 (2): 1289-93.

[8] Hamlat, A., Casallo-Quilliano, C., Saikali, S., Lesimple, T., and Brassier, G. 2004. "Epithelioid Haemangioendothelioma of the Infundibular-Hypothalamic Region: Case Report and Literature Review." J. Neurooncol. 67 (2): 361-6.

[9] Di Girolamo, A., Giacomini, P. G., Coli, A., Castri, F., de Padova, A., and Bigotti, G. 2003. "Epithelioid haemangioendothelioma Arising in the Nasal Cavity." $J$. Laryngol. Otol. 117 (1): 75-7.

[10] Molina Palma, M. I., Cervantes Gongora, J. A., Garcia de la Torre, E., Conde Perez de la Blanca, I., and Ramirez Tortosa, C. L. 2002. "Primary Intraoral Epithelioid Haemangioendothelioma. Case Report and Review of the Literature." Acta Otorrinolaringol Esp. 53 (1): 215-8.

[11] Weiss, S. W., and Goldblum, J. R. 2001. Enzinger and Weiss's Soft Tissue Tumors. 4th ed. St. Louis. Mo: Mosby: 891-900.

[12] Sardaro, A., Bardoscia, L., Petruzzelli, M. F., and Portaluri, M. 2004. "Epithelioid Haemangioendothelioma: An Overview and Update on a Rare Vascular Tumour." Oncology Reviews 8 (1): 82-91.

[13] Hamirani, Y. S., Ndigwe, E., Kompella, S., Mannuel, H., Shekitka, K., Seibel, J., and Esquivel, J. 2008. Epithelioid Haemangioendothelioma of the Peritoneum. Assessed February 10, 2017. http://www.mdmag.com/journals/resident-and-staff/2008/ 2008-04/2008-04_03.

[14] Naqvi, J., Ordonez, N. G., Luna, M. A., Williams, M. D., Weber, R. S., and El-Naggar, A. K. 2008. "Epithelioid Haemangioendothelioma of the Head and Neck: Role of Podoplanin in the Differential Diagnosis." Head Neck Pathol. 2 (2): 25-30.

[15] Zarifa, Y., and Kemal, K. 2014. "CAMTA Immunostaining Is not Useful in Differentiating Epithelioid Haemangioendothelioma from Its Potential Mimickers." Tjpath. 30 (1): 159-65.

[16] Läuffer, J. M., Zimmermann, A., Krähenbühl, L., Triller, J., and Baer, H. U. 1996. "Epithelioid Haemangioendothelioma of the Liver: A Rare Hepatic Tumor." Cancer 78 (1): 2318-27.

[17] Makhlouf, H. R., Ishak, K. G., and Goodman, Z. D. 1999. "Epithelioid Haemangioendothelioma of the Liver: A Clinicopathologic Study of 137 Cases." Cancer 85 (1): 562-82. 